\title{
A web-based system for supporting collaboration towards resolving oncology issues
}

\author{
NIKOS KARACAPILIDIS ${ }^{1}$ and DIMITRIOS KOUKOURAS ${ }^{2}$ \\ ${ }^{1}$ IMIS Lab, MEAD and ${ }^{2}$ Medical School, University of Patras, 26500 Rio-Patras, Greece
}

Received July 7, 2005; Accepted October 10, 2005

\begin{abstract}
The coordinated consideration of diverse perspectives and knowledge sources in medical decisionmaking contexts is undoubtedly of crucial importance, in that it augments the quality of both the overall process and decisions to be made. Towards this end, this study presents a web-based system that aims at the exploitation of personalized knowledge through a structured process of collaborative and argumentative resolution of issues raised in such settings. By using the system, a consensus emerges through the process of collaboratively considering alternative interpretations of the issue under consideration. Using an illustrative example of collaboration between a group of experts deciding on the most appropriate treatment for a particular case of breast cancer, we present the design rationale, features and functionality of the proposed system. Much attention is also paid to the associated knowledge management issues. The system can be used for distributed, asynchronous collaboration, allowing users to surpass the requirements of working at the same place and time.
\end{abstract}

\section{Introduction}

Medical decision making is associated with a plethora of rigorous and systematic approaches, which traditionally build on the fundamentals of decision analysis and theory, economic evaluation and evidence-based quality assessment, aim at improving the health and clinical care of individuals and assist with the development of health policy. It is usually carried out by a group of experts acting collaboratively. In such settings, conflicts of interest are inevitable; thus, support for achieving a consensus and compromise is required. When dealing with a medical issue, each decision maker (medical

Correspondence to: Dr Nikos Karacapilidis, Industrial Management and Information Systems Lab, Department of Mechanical Engineering and Aeronautics, University of Patras, GR 26500 Rio-Patras, Greece E-mail: nikos@mech.upatras.gr

Key words: medical decision making, electronic collaboration, knowledge management, argumentation, web-based systems, breast cancer doctor, paramedic, etc.) may adopt and consequently suggest his/her own opinion, which may address goals at a specific level. Opinions may differ about the relevance or value of a position when resolving an issue. Moreover, decision makers may have arguments that support or discourage alternative solutions. In addition, one must often confront the existence of insufficient or too much information; for some parts of the problem, useful relevant information for making a decision is missing, while the time needed for the retrieval and comprehension of the existing volume of information is prohibitive for others. Furthermore, factual knowledge is not always sufficient for making a decision; value judgments, depending on the role and the goals of each decision maker, are among the critical issues requiring attention. Finally, decision makers are not necessarily proficient in computer science and information technology, and need the appropriate tools to easily follow and participate in the associated processes. Such tools should stimulate their participation and give them an active role.

From a technical point of view, the potential exploitation of information and communication technologies in developing computer-based systems that assist collaborative medical decision making is obvious. Such systems fall in the broad category of collaborative decision support systems (CDSSs), which have been defined as interactive systems that facilitate the solution of ill-structured problems by a set of decision makers working together as a team (1). The main objective of a CDSS is to augment the effectiveness of decision groups through the interactive sharing of information between group members and the computer. This can be achieved by removing communication impediments, and providing techniques for structuring the decision analysis and systematically directing the pattern, timing, or content of the related discussions $(2,3)$. Major issues arising during the development of such a system are the effective work organization to improve coordination and the use of communication technology to make decision making more efficient. The provision of rules and procedures for achieving consistency and automation of data processing, especially in data intensive decision-making situations, are also of high importance.

At the same time, the role and value of knowledge management in diverse decision-making settings is continuously emphasized. It is argued that the most strategically important feature of an organization is its body of collective knowledge (4). Such knowledge resides in an evolving set of assets including the employees, structure, culture and processes of 
the organization. Employee knowledge, particularly tacit knowledge, is identified as dominant, which is decisive at all mental levels and must be fully exploited (5). Such exploitation refers to the transformation of tacit knowledge to codified information, which is considered a core process (6). The above advocate the adoption of a knowledge-based decision-making view (7). Accordingly, decisions should be considered pieces of descriptive or procedural knowledge referring to an action commitment. In this way, the decision-making process is able to produce new knowledge, such as evidence that justifies or challenges an alternative or practice to be followed or avoided after evaluating a decision, thus providing a refined understanding of the problem. On the other hand, the knowledge base of facts and routines is altered in a decisionmaking context, since it must reflect the ever-changing external environment and internal structures of an organization (8). For all of these reasons, knowledge management activities such as knowledge elicitation, representation and distribution, may significantly influence the creation of decision models to be adopted, thus enhancing the decision-making process (9).

Taking into account the above requirements, this study presents a web-based system that aims at the exploitation of personalized knowledge through a structured process of collaborative and argumentative resolution of issues raised in medical decision-making settings. The proposed system can be used for distributed, asynchronous collaboration, allowing users to surpass the requirements of working at the same place and time. Its features and functionality are demonstrated through an illustrative example of collaboration between a group of medical doctors deciding on the most appropriate treatment for a particular case of breast cancer.

\section{Materials and methods}

Background issues. Medical decision making is a complex process that involves assessing and analyzing patient information, diagnostic data and scientific evidence to make a diagnosis and select the appropriate treatment. The application of appropriate decision models provides a valuable framework for guidance and systematic analysis to assist physicians with the complexity of medical decision making and reasoning (10). Computer-based medical decision support systems have been defined as 'any software designed to directly aid in clinical decision making in which characteristics of individual patients are matched to a computerized knowledge base for the purpose of generating patient-specific assessments or recommendations that are then presented to clinicians for consideration' $(11,12)$. Such systems have been successfully used for diverse applications such as prevention of sudden cardiac death $(13,14)$, diagnosis and treatment of infectious diseases in intensive care units (15), and pediatric cancer subtype identification (16). Medical expert systems consist of an early and broadly used category of computer-based medical decision support systems. They provide pre-selected rules (in the form of 'if-then-else') for decision-making within specialized domains of knowledge, but are limited by the fixed choices and date of the expert opinions embodied in the decision rules (17). Modern methods (model-based and black-box methods) of representing medical reasoning include production rules, Bayesian probabilistic methods, neural networks, case-based reasoners, decision trees, stochastic trees, support vector machines, and GRID environments $(15,16,18-21)$, among others.

The above approaches have been criticized for limiting physician choices to the 'expert writing the program' (17) and not being 'intuitive to the human cognitive processes' (16). To remedy these problems, new formalisms based on the behavior of people when acting and communicating have been proposed (22). These representation schemes have been founded along two directions: i) the human activity system models of Checkland's soft systems methodology (23) to accommodate the 'softness' of organizational life; and ii) the language/action perspective (24) and the Speech Act theory (25) to emphasize the conversational nature of humancentered organizational activity. The latter consider the utterance of various types of communicative actions as the backbone of business process models $(26,27)$. In both cases, related structures and methodologies concentrate on the representation of knowledge (content), but barely consider knowledge creation through interaction.

As far as interaction is concerned, ICT infrastructure to support people working in teams has been a subject of interest for a long time (28). Such systems aim at facilitating group decision-making processes by providing forums to express opinions, as well as qualitative and quantitative tools for aggregating proposals and evaluating their impact on the issue at hand $(29,30)$. Current systems exploit intranet or Internet technologies to connect decision makers in a way that encourages dialogue and stimulates the exchange of knowledge (enhancing 'knowing' rather than 'knowledge'). The more recent computer-based Knowledge Management System (KMS) intends on providing a corporate memory, an explicit disembodied and persistent representation of the knowledge and information in an organization, as well as mechanisms that improve the sharing and dissemination of knowledge by facilitating interaction and collaboration among the parties involved (9). Compared to problem structuring methodologies and tools, they lack a concrete theoretical basis, as well as methodological support with respect to social interaction.

The proposed system. Taking into account the above issues, we have implemented a web-based system that supports the collaboration conducted in a medical decision making context by facilitating the creation, leveraging and utilization of relevant knowledge. We have followed an argumentative reasoning approach, which extends the one conceived in the development of the Hermes system (31), by providing additional knowledge management and decision-making features. According to our approach, discourses about complex problems in a health unit are considered as social processes and, as such, result in the formation of groups whose knowledge is clustered around specific views of the problem. Following an integrated approach, we have developed a system that provides experts engaged in such a discourse with the appropriate means to collaborate towards the solution of diverse issues. In addition to providing a platform for group reflection and capturing organizational memory, our approach augments teamwork in terms of knowledge elicitation, sharing and construction, thus enhancing the quality of the 


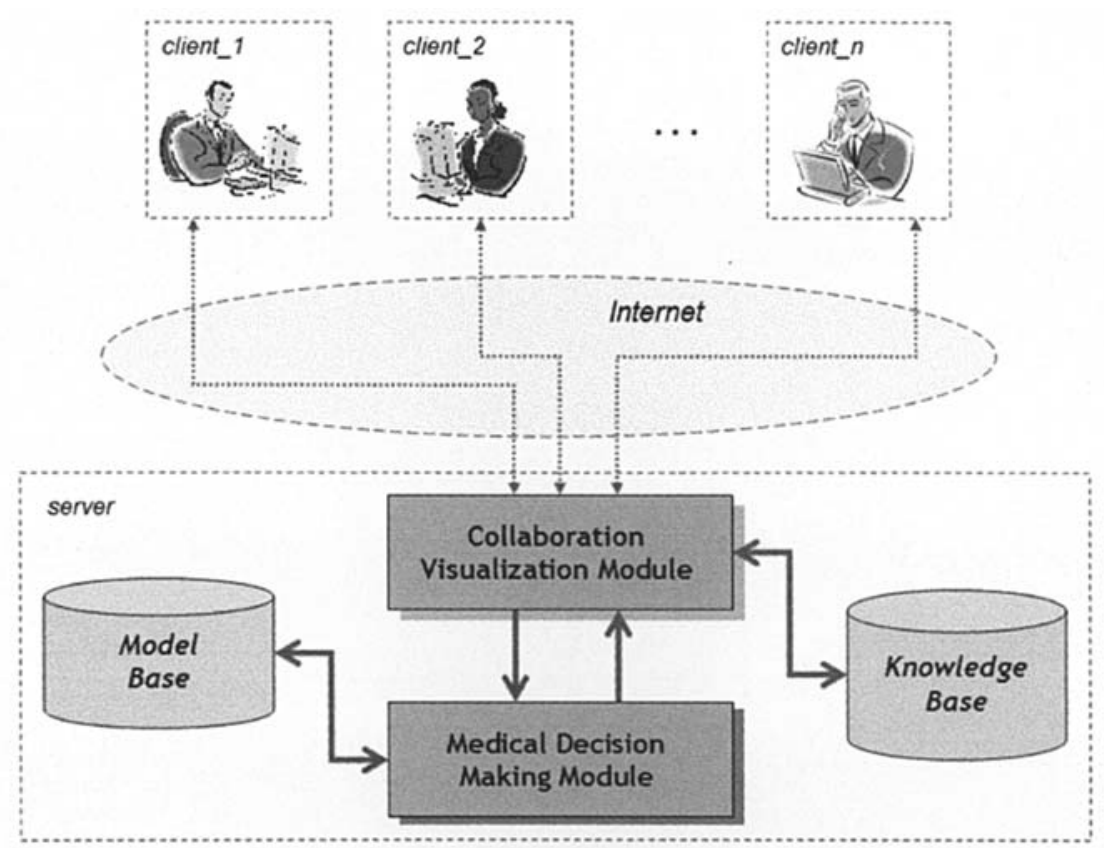

Figure 1. Architecture of the proposed system.

overall process. This is due to its structured language for conversation and its mechanism for evaluating alternatives. Taking into account the input provided by individual experts, the system constructs an illustrative discourse-based knowledge graph that is composed of ideas expressed so far, and their supporting documents. Such documents may contain diverse types of multimedia clinical data, such as patient history, biopsy results, medical images, etc. Moreover, through the integrated decision support mechanisms, experts are continuously informed about the status of each discourse item and can reflect further according to their beliefs and interests on the outcome of the discussion. In addition, our framework aids group sense-making and mutual understanding through the collaborative identification and evaluation of diverse opinions.

As shown in Fig. 1, our system builds on a server-client network architecture and is composed of two basic components, namely the collaboration visualization and medical decisionmaking modules. The former provides a shared web-based workspace for storing and retrieving messages and documents deployed by participants of the discussion (experts and/or members of a health unit), using the widely accepted XML document format. It also provides the interface through which participants can connect with the system via Internet using a standard web browser (installation of specific software to use the system is unnecessary). Exploitation of the web platform means low operational costs and easy access to the system. The knowledge base of the system maintains all of the above items (messages and documents), which may be considered, appropriately processed and transformed, or re-used in future discussions. The storage of documents and messages in an ongoing discussion takes place automatically upon their insertion in the discussion. On the other hand, the retrieval of knowledge is performed through appropriate interfaces that aid participants in exploring the contents of the knowledge base and exploit previously stored or generated knowledge for their current needs. Thus, our approach builds a 'collective memory' of a health unit community. On the other hand, the medical decision-making module is responsible for the reasoning and evaluation purposes of the system. Alternative mechanisms for these purposes can be invoked according to the need of the participant and the context under consideration. These mechanisms follow well-defined and broadly accepted algorithms based on diverse decision-making approaches (such as multi-criteria decision making, argumentation-based reasoning, utility theory, risk assessment, etc.), which are stored in the model base of the system.

The basic discourse elements in our system are issues, alternatives, positions, and preferences. Issues correspond to problems to be solved, decisions to be made, or goals to be achieved, which are intiated by members of a health unit and are open to dispute (the root entity of a discourse-based knowledge graph has to be an issue). For each issue, users may propose alternatives (i.e. solutions to the problem under consideration) that correspond to potential choices. Nested issues, in cases where some alternatives need to be grouped, are also allowed. Positions are asserted to support the selection of a specific course of action (alternative), or avert user interest by expressing an objection. A position may also refer to another previously asserted position, thus arguing in favor or against it. Finally, preferences provide individuals with a qualitative way to weigh reasons for and against the selection of a certain course of action. A preference is a tuple of the form (position, relation, position), where the relation can be 'more important than' or 'of equal importance to' or 'less important than.' The use of preferences results in the assignment of various levels of importance to the alternatives at hand. Like other discourse elements, they are subject to further argumentative discussion.

These four types of elements enable users of the system to contribute their knowledge to a particular medical problem by entering issues, alternatives and positions, and to express their relevant values, interests and expectations by entering positions and preferences. The system therefore supports 


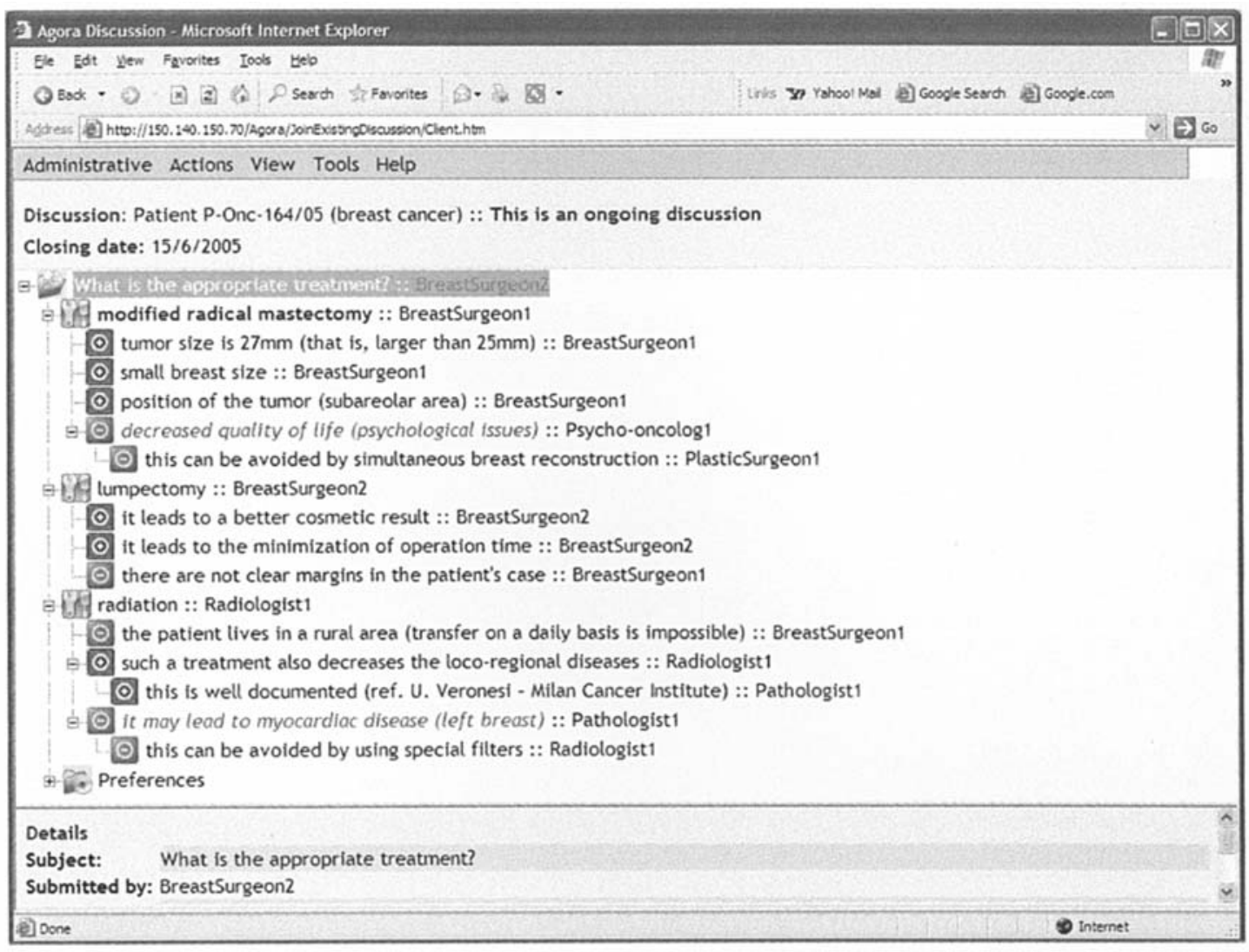

Figure 2. An instance of discourse about the appropriate treatment for a patient with breast cancer.

both the rationality-related and social dimensions of the medical decision-making process. Moreover, the system continuously processes elements entered by the users (by triggering its reasoning mechanisms) each time a new element is entered in the graph, thus facilitating users to become aware of elements for which there is (or not) sufficient (positive or negative) evidence, and accordingly conduct the discussion to reach a consensus.

An example of use. We present the features and functionalities of the proposed system in more detail, and its applicability in supporting collaboration in a medical decision-making context, through an illustrative example of collaboration between a group of experts deciding on the appropriate treatment for a patient with breast cancer. The group for the particular case consisted of two breast surgeons, a plastic surgeon, a pathologist, a psycho-oncologist and a radiologist. Participants were geographically dispersed and had access to the system via an Internet connection and their favorite web browser. It must be noted that beyond having access to the system, one needs to be authorized to either view only and/or participate in a specific collaborative discourse, which can be set and facilitated through the system. Such rights are given by the initiator or moderator of a discourse through appropriate interfaces of the system. One may have access to a discourse and view the related items, but not be allowed to contribute. In our example, all participants had full rights to the specific discourse and sufficient familiarity with using computers and the Internet. Less than $2 \mathrm{~h}$ training, aimed at explaining the rationale, features and functionalities of the system, was administered before participants used the system for the first time.
Fig. 2 depicts the basic interface of the system (i.e. discussion graph) for an instance of the collaboration conducted. Our approach maps the overall collaboration process to a discourse-based knowledge graph with a hierarchical structure. Each entry in the graph corresponds to an argumentation element (issue, alternative, position or preference), and each element is accompanied by an icon that indicates the element type. There are also icons for folding/ unfolding purposes, thus enabling users to concentrate on a specific part of the graph; this is particularly useful for graphs of considerable length and complexity. Each entry in the graph may contain the user-name of the person who made the submission and the date (the appearance of each entry can be changed using options provided under the View menu; two different formats are shown in Figs. 2 and 3). In the example discussed in this study, the user-names correspond to the expertise of participants (names are disguised for confidentiality). The lower pane of the window shown in Fig. 2 provides more details about a selected entry of the discussion graph (users can select an entry by clicking on it).

In our case, the overall issue under discussion is 'What is the appropriate treatment,' while the three alternatives, of 'modified radical mastectomy,' 'lumpectomy' and 'radiation' have been asserted so far by the users BreastSurgeon1, BreastSurgeon2 and Radiologist1, respectively. The users have argued extensively by expressing positions speaking in favor or against them. For example, 'position of the tumor (subareolar area)' is a position asserted by BreastSurgeon1 that argues in favor of the first alternative, and 'it may lead to myocardiac disease (left breast)' is a position asserted by Pathologist 1 that argues against the third alternative. All 


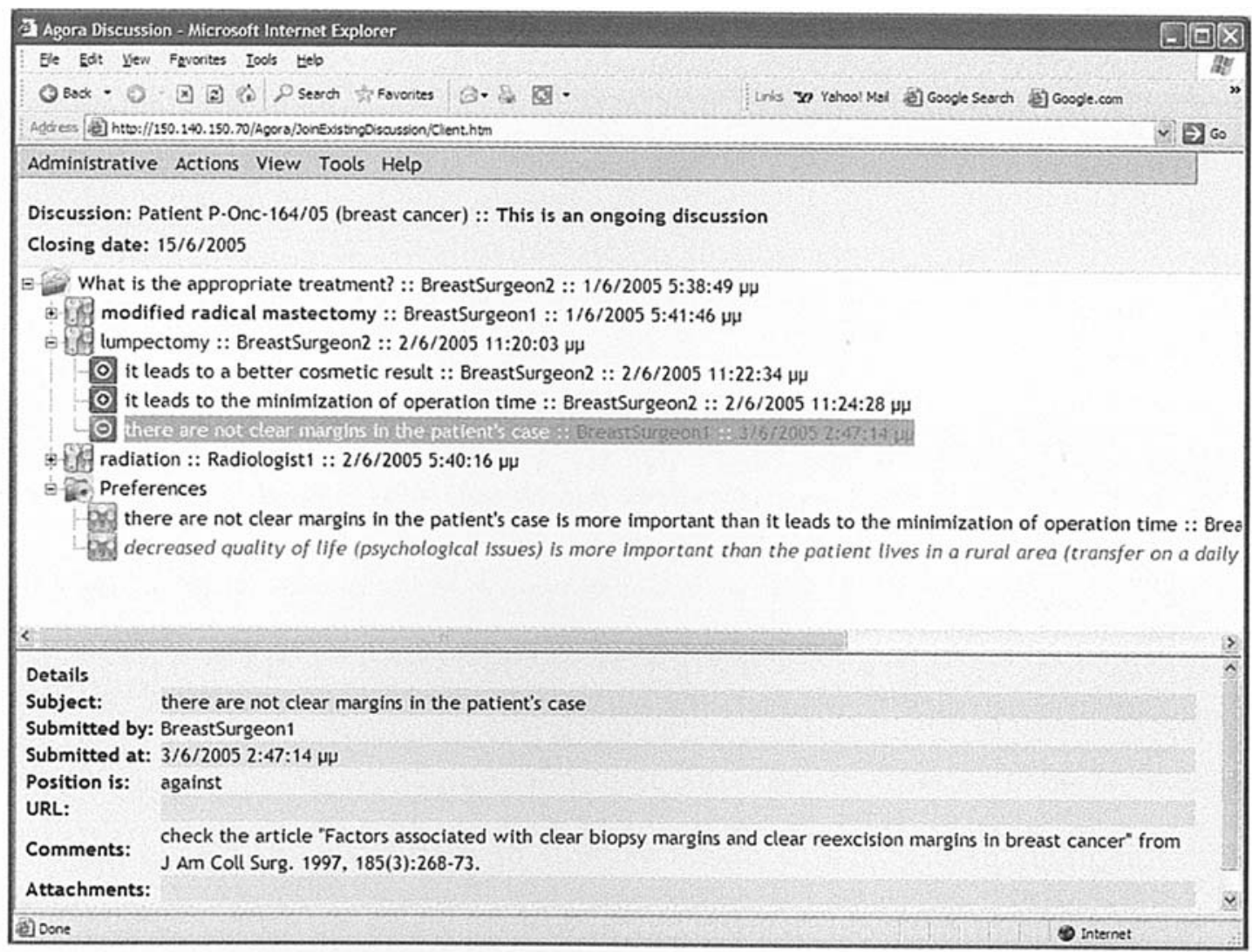

Figure 3. Another instance of the same discourse.

graph entries are subject to multi-level argumentation. For instance, 'this is well documented (ref. U. Veronesi - Milan Cancer Institute)' has been asserted by Pathologist1 to further validate the 'such a treatment also decreases the loco-regional diseases' position asserted by Radiologist1, and 'this can be avoided by simultaneous breast reconstruction' challenges the 'decreased quality of life (psychological issues).'

As noted above, users may also assert preferences about previously expressed positions. At the bottom of the main pane in Fig. 3, user BreastSurgeon1 has expressed a preference concerning the relative importance between the positions 'there are not clear margins in the patient's case' and 'it leads to the minimization of operation time,' arguing that the first position is of bigger importance to him; user BreastSurgeon2 has expressed a second preference in the instance shown. Users may also express arguments in favor or against a preference.

When selecting a discourse item by clicking on it, detailed information about this item is provided in the lower pane of the basic interface of the system. In Fig. 3, the details concern the position 'there are not clear margins in the patient's case.' These comprise information about the user who submitted the selected discussion element, its submission date, any comments that the user may have inserted, as well as links (URLs) to related web pages and multimedia documents (containing diverse types of clinical data) uploaded by the user to the system in order to justify this element and aid his/her peers in their contemplation. Thus, the system offers users the capability of exchanging oncological data in an implicit way (data can be viewed by anyone participating in a discussion, and are stored in the knowledge base of the system).
Further to the argumentation-based structuring of a discourse, the system integrates a reasoning mechanism that determines the status of each discussion entry, with the ultimate aim of keeping users aware of the discourse outcome. More specifically, alternatives, positions and preferences of a graph have an activation label indicating their current status, which can be 'active' or 'inactive' (inactive entries appear in red italics) (Fig. 2). This label is calculated according to the argumentation underneath and the type of evidence specified. In Fig. 2, the position 'decreased quality of life (psychological issues)' is inactive because, according to the argumentation rule for this specific discussion, it has been defeated by the position 'this can be avoided by simultaneous breast reconstruction' [the position 'it may lead to myocardiac disease (left breast)' is inactive for the same reason]. Activation in our system is a recursive procedure; a change in the activation label of an element is propagated upwards in the discussion graph. Depending on the status of positions and preferences, the mechanism goes through a scoring procedure for the alternatives of an issue. A detailed presentation of more technical details concerning the argumentation-based reasoning and scoring mechanisms of the system can be found in a previous study (31). At each discussion instance, the system informs users of the most prominent alternative solution (shown in blue bold font) according to the underlying argumentation. In Figs. 2 and 3, 'modifies radical mastectomy' is the better justified solution so far (all items asserted under the first and third alternatives are folded in Fig. 3 for space reasons). However, this may change depending on the type of future arguments. In other words, each time an alternative is introduced to the discussion, the issue in which it belongs is 
updated, and another solution may be indicated by the system.

The system also integrates e-mail and electronic messaging features, which are options provided under the Tools menu, to further facilitate the communication among users before one asserts an argumentation element in the graph. Users may therefore exchange oncological data in an explicit way. Although these data aid contemplation during a discourse and may become public upon the appropriate assertion of a discourse element in the discussion graph as described above, they cannot be viewed by anyone participating in a discussion. The insertion of all types of elements in the graph is performed through appropriately designed interfaces deployed upon user selection under the Actions menu. Such functions include the opening of an issue, insertion of a new alternative to an issue, insertion of a new position in favor or against an existing position, preference or alternative, and insertion of a new preference to an existing issue.

\section{Results}

The proposed system has been evaluated with more than 30 pilot and real discussions to estimate its perceived usefulness and ease of use. These discussions were initiated by physicians working at the Oncology Units of two different hospitals, while the types of cases examined concerned contemplation among medical experts on the appropriate treatment for a specific patient (diverse oncological problems have been considered). In these discussions, a human moderator supervised the argumentation and assisted users whenever needed. The role of the supervisor was similar to that of a system administrator, i.e. provide access rights, ensure that elements were inserted to the right position in the discussion graph, etc. Medical experts used the system from remote machines, mostly in an asynchronous way, to express and validate their proposals and argue those of others. Evaluation of the system was conducted through interviews and questionnaires aimed at identifying how well the system structures a medical decision-making discussion, whether it stimulates interaction and discussion among individuals, how easy it is to learn and get acquainted with the system, how easy it is to participate in electronic argumentation, whether or not the system is enjoyable, and whether individuals intend to use it again. A second group of questions were related to the interfaces of the system, asking users to evaluate its ease of use in finding, understanding and using the available options of the interfaces and navigating the system, understanding the content of electronic argumentation, and whether their past and future actions were clear for the duration of electronic argumentation.

Feedback received from the evaluation of the system is encouraging (for detailed evaluation results from a first version of the system, see ref. 31). It has been concluded that users found the system to be useful, and easy to learn and use its basic functionality. The general attitude towards using the system was also positive, which makes users want to use it again in similar discussions and contexts. However, there were some difficulties in getting acquainted with an argumentation session conducted via an electronic channel (i.e. Internet). Users observed that such an experiment is different from the usual and familiar physical argumentation that takes place in face-to-face meetings. In general, electronic argumentation is more demanding than the physical one, requiring participants to concisely express each new element (short text), then associate it with a previously asserted element by the same or different user. Moreover, users must be able to comprehend the concisely expressed elements asserted by their peers. A longer training period could remedy these difficulties.

In any case, the most positive feedback we received from the majority of users was that the proposed approach leads to an improvement of both the quality of a medical decisionmaking process and the decisions to be taken. Thus, its adoption results in enhanced patient treatment. Moreover, the exchange and elaboration of individual knowledge through the system features and functionalities have been acknowledged as significant factors towards improving learning in a health unit.

\section{Discussion}

Quoting Davenport and Prusak, 'knowledge is a fluid mix of framed experience, values, contextual information, and expert insight that provides a framework for evaluating and incorporating new experiences and information (32).' The above definition perfectly suits the case of medical decision making and has influenced the design of the system described in this study, which aims at the exploitation of personalized knowledge through a structured process of collaborative and argumentative resolution of issues raised in a medical decisionmaking setting. By using the system, consensus emerges through the process of collaboratively considering alternative understandings of the issue under consideration. It has been widely argued that the visualization of argumentation conducted by a group of experts working collaboratively towards solving a problem can facilitate the overall process in many ways, such as explicating and sharing individual representations of the problem, maintaining focus on the overall process, maintaining consistency and increasing plausibility and accuracy (33). It also enhances the collective knowledge of the group.

The direction of future research concerns the integration of additional functionalities to the proposed system. Factbased decision-making must also be elaborated through the exploitation of past cases and related discussions. Moreover, we intend to provide participants of a specific discourse with the ability to evaluate its outcome after a decision has been made and the associated treatment has been followed. Users of the system will also be able to check if decisions made on previous cases were correct or not, and act accordingly. Finally, research needs to be performed towards defining the ontologies that will best accommodate the proposed integration of knowledge management and decision-making processes in a medical decision-making setting.

\section{References}

1. Kreamer KL and King JL: Computer-based systems for cooperative work and group decision making. ACM Computing Surveys 20: 115-146, 1988.

2. Dennis AR, George JF, Jessup LM, Nunamaker JF Jr and Vogel DR: Information technology to support electronic meetings. MIS Quarterly 12: 591-624, 1988. 
3. Nunamaker JF Jr, Applegate L and Konsynski B: Facilitating group creativity: experience with a group decision support system. J Management Info Systems 3: 5-19, 1987.

4. Spender J: Organizational knowledge, learning and memory: three concepts in search of a theory. J Organization Change Management 9: 63-78, 1996.

5. Nonaka I: A dynamic theory of organizational knowledge creation. Organization Sci 5: 14-37, 1994.

6. Cohendet P and Steinmueller WE: The codification of knowledge: a conceptual and empirical exploration. Industrial Corporate Change 9: 195-209, 2000

7. Holsapple CW and Whinston AB: Decision Support Systems: A Knowledge-Based Approach. West Publishing Company, St. Paul, MN, 1996.

8. Bhatt $\mathrm{G}$ and Zaveri J: The enabling role of decision support systems in organizational learning. Decision Support Systems 32: 297-309, 2002

9. Bolloju N, Khalifa M and Turban E: Integrating knowledge management into enterprise environments for the next generation decision support. Decision Support Systems 33: 163-176, 2002.

10. Sanders GD, Nease RF and Owens DK: Publishing web-based guidelines using interactive decision models. J Eval Clin Practice 7: 175-189, 2001

11. Durieux P, Nizard R, Ravaud P, Mounier N and Lepage E: A clinical decision support system for prevention of venous thromboembolism. JAMA 283: 2816-2821, 2000.

12. Hunt DL, Haynes RB, Hanna SE and Smith K: Effects of a computer-based clinical decision support system on physician performance and patient outcome: a systematic review. JAMA 280: 1339-1346, 1989

13. Sanders GD, Nease RF and Owens DK: Design and pilot evaluation of a system to develop computer-based site-specific clinical-practice guidelines from decision models. Med Decision Making 20: 145-159, 2000.

14. Sanders GD, Hagerty CG, Sonnenberg FA, Hlatky MA and Owens DK: Distributed decision support using a web-based interface: prevention of sudden cardiac death. Med Decision Making 19: 157-166, 1999.

15. Schurink CAM, Lucas PJF, Hoepelman IM and Bonten MJM: Computer-assisted decision support for the diagnosis and treatment of infectious diseases in intensive care units. Lancet Infectious Diseases 5: 305-312, 2005.

16. Tung WL and Quek C: GenSo-FDSS: a neural-fuzzy decision support system for pediatric ALL cancer subtype identification using gene expression data. Artif Intell Med 33: 61-88, 2005.

17. Frenster JH: Expert systems and open systems in medical artificial intelligence. Proc Am Med Systems Informatics Congress 7: 118-120, 1989.

18. Arocha JF, Wang D and Patel VL: Identifying reasoning strategies in medical decision making: a methodological guide. J Biomed Informatics 38: 154-171, 2005.
19. Handler JA, Feied CF, Coonan K, Vozenilek J, Gillam M, Peacock PR, Sinert R and Smith MS: Computerized physician entry and online decision support. Acad Emerg Med 11: 1135-1141, 2004.

20. Hazen GB: Stochastic Trees and the StoTree Modeling Environment: Models and Software for Medical Decision Analysis. Northwest University, 2001.

21. Blanquer I, Hernandez V, Segrelles D, Robles M, Garcia JM and Robledo JV: Clinical Decision Support Systems (CDSS) in GRID Environments. Stud Health Tech Info 112: 80-89, 2005.

22. Van Reijswoud VE, Mulder H and Dietz J: Communicative action-based business process and information systems modelling with DEMO. Info Systems J 9: 117-138, 1999.

23. Checkland P and Scholes J: Soft Systems Methodology in Action. John Wiley and Sons, Chichester, 1990.

24. Dignum F, Dietz J, Verharen E and Weigand H (eds.): The Language/Action Perspective: Proceedings of the 1st International Workshop on Communication Modeling, Electronic Workshops in Computing, Springer-Verlag, Berlin, 1996.

25. Searle JR: Speech Acts: An Essay in the Philosophy of Language. Cambridge University Press, Cambridge, 1969.

26. Medina-Mora R, Winograd T, Flores R and Flores F: The Action Workflow Approach to Workflow Management Technology. In: Proceedings of the 4th Conference on Computer Supported Cooperative Work, October 31-November 4, Toronto, Canada. ACM Press, New York, 1992

27. Winograd T and Flores F: Understanding Computers and Cognition: A New Foundation for Design. Ablex, Norwood, NJ, 1986.

28. Fjermestad J and Hiltz SR: Group support systems: a descriptive evaluation of case and field studies. J Management Info Systems 17: 115-159, 2000.

29. Bose R: Group support systems: technologies and products selection. Industrial Management Data Systems 103: 649-656, 2003.

30. Cummings JN: Work groups, structural diversity and knowledge sharing in a global organization. Management Sci 50: 352-364, 2004.

31. Karacapilidis N and Papadias D: Computer supported argumentation and collaborative decision making: The HERMES system. Info Systems 26: 259-277, 2001.

32. Davenport T and Prusak L: Working Knowledge. Harvard Business School Press, Boston, MA, 1998.

33. Kirschner P, Buckingham Shum S and Carr C: Visualizing Argumentation: Software Tools for Collaborative and Educational Sense-Making. Springer Verlag, London, 2003. 